Bull. Korean Math. Soc. 50 (2013), No. 5, pp. 1693-1710

http://dx.doi.org/10.4134/BKMS.2013.50.5.1693

\title{
MULTIPLICITY OF SOLUTIONS FOR BIHARMONIC ELLIPTIC SYSTEMS INVOLVING CRITICAL NONLINEARITY
}

\author{
Dengfeng Lü and Jianhai XiaO
}

ABstract. In this paper, we consider the biharmonic elliptic systems of the form

$$
\begin{cases}\Delta^{2} u=F_{u}(u, v)+\lambda|u|^{q-2} u, & x \in \Omega, \\ \Delta^{2} v=F_{v}(u, v)+\delta|v|^{q-2} v, & x \in \Omega, \\ u=\frac{\partial u}{\partial n}=0, v=\frac{\partial v}{\partial n}=0, & x \in \partial \Omega,\end{cases}
$$

where $\Omega \subset \mathbb{R}^{N}$ is a bounded domain with smooth boundary $\partial \Omega, \Delta^{2}$ is the biharmonic operator, $N \geq 5,2 \leq q<2^{*}, 2^{*}=\frac{2 N}{N-4}$ denotes the critical Sobolev exponent, $F \in C^{1}\left(\mathbb{R}^{2}, \mathbb{R}^{+}\right)$is homogeneous function of degree $2^{*}$. By using the variational methods and the Ljusternik-Schnirelmann theory, we obtain multiplicity result of nontrivial solutions under certain hypotheses on $\lambda$ and $\delta$.

\section{Introduction}

The main purpose of this paper is to study the multiplicity of nontrivial solutions for the following biharmonic elliptic system:

$$
\begin{cases}\Delta^{2} u=F_{u}(u, v)+\lambda|u|^{q-2} u, & x \in \Omega, \\ \Delta^{2} v=F_{v}(u, v)+\delta|v|^{q-2} v, & x \in \Omega, \\ u=\frac{\partial u}{\partial n}=0, v=\frac{\partial v}{\partial n}=0, & x \in \partial \Omega,\end{cases}
$$

where $\Omega \subset \mathbb{R}^{N}(N \geq 5)$ is a bounded domain with smooth boundary $\partial \Omega$, $\Delta^{2}$ is the biharmonic operator, $2 \leq q<2^{*}, 2^{*}=\frac{2 N}{N-4}$ denotes the critical Sobolev exponent, $F \in C^{1}\left(\mathbb{R}^{2}, \mathbb{R}^{+}\right)$is homogeneous function of degree $2^{*}$, $\left(F_{u}(u, v), F_{v}(u, v)\right)=\nabla F, \frac{\partial}{\partial n}$ is the outer normal derivative, and $\lambda, \delta$ are positive parameters.

The starting point on the study of the system (1.1) is its scalar version:

$$
\begin{cases}\Delta^{2} u=|u|^{2^{*}-2} u+\lambda|u|^{q-2} u, & x \in \Omega \\ u=\frac{\partial u}{\partial n}=0, & x \in \partial \Omega\end{cases}
$$

Received December 5, 2012.

2010 Mathematics Subject Classification. 35J50, 35B33.

Key words and phrases. biharmonic elliptic system, critical Sobolev exponent, variational method, multiple solutions. 
The interest of problem (1.2) grew from its resemblance to some nonlinear equations arising from a geometric context and which have extensively been studied for various $q \in\left(1,2^{*}\right)$ in recent years. Many important results were obtained in these publications (see $[3,5,7,9,10,11,18,21,23]$ and the references therein). For example, Edmunds, et al. [7] showed that, if $q=2$, the equation (1.2) has a nontrivial solution provided $N \geq 8$ and $0<\lambda<\lambda_{1}$, where $\lambda_{1}$ is the first eigenvalue of the operator $\left(\Delta^{2}, H_{0}^{2}(\Omega)\right)$. Recently, Zhang [23] obtained the existence of one positive solution of equation (1.2) with the sublinear perturbation of $1<q<2$ and under the Navier boundary condition.

In recent years, more and more attention has been paid to the elliptic systems. In particular, Hsu and Lin in [13] concerned the case $F(u, v)=2|u|^{\alpha}|v|^{\beta}$, $\alpha>1, \beta>1$ satisfying $\alpha+\beta=\frac{2 N}{N-2}$, i.e., the following elliptic system

$$
\begin{cases}-\Delta u=\frac{2 \alpha}{\alpha+\beta}|u|^{\alpha-2} u|v|^{\beta}+\lambda|u|^{q-2} u, & x \in \Omega \\ -\Delta v=\frac{2 \beta}{\alpha+\beta}|u|^{\alpha}|v|^{\beta-2} v+\delta|v|^{q-2} v, & x \in \Omega \\ u=v=0, & x \in \partial \Omega\end{cases}
$$

Using the Nehari manifold method, the authors in [13] obtained the existence of two positive solutions of system (1.3) with the sublinear perturbation of $1<q<$ 2. Han in [12] using the variational theory and the Ljusternik-Schnirelmann category theory has proved that system (1.3) has at least cat $\Omega(\Omega)$ positive solutions if $\lambda, \delta \in\left(0, \lambda^{*}\right)$, where $0<\lambda^{*}<\lambda_{1}$, and $\lambda_{1}$ is the first eigenvalue of $\left(-\Delta, H_{0}^{1}(\Omega)\right)$. Furthermore, Ding and Xiao [6] extended the result in [12] to the $p$-Laplacian case and obtained similar result.

In this paper, we complement and extend the results of $[6,12,13]$ to the biharmonic critical case and $2 \leq q<2^{*}$. Considering the multiplicity of nontrivial solutions of problem (1.1), we show that problem (1.1) has at least $\operatorname{cat}_{\Omega}(\Omega)$ nontrivial solutions when the pair of parameters $\lambda, \delta$ satisfied a certain condition. Our main tool here is the Ljusternik-Schnirelmann category theory (see $[1,19]$ ), but we had to overcome several technical difficulties that appeared, for example, when treating a more general critical term like $F(u, v)$. To the best of our knowledge, problem (1.1) has not been considered before. Thus it is necessary for us to investigate the critical biharmonic elliptic system (1.1) deeply. We also refer to more related systems, which can be seen in $[2,8,14,15,16,17,20]$ and references therein.

Before stating our results, we need the following assumptions:

$\left(F_{0}\right) F \in C^{1}\left(\mathbb{R}^{2}, \mathbb{R}^{+}\right)$and $F(t u, t v)=t^{2^{*}} F(u, v)(t>0)$ holds for all $(u, v) \in \mathbb{R}^{2}$; $\left(F_{1}\right) F(u, 0)=F(0, v)=F_{u}(u, 0)=F_{v}(0, v)=0$, where $u, v \in \mathbb{R}$;

$\left(F_{2}\right) F_{u}(u, v), F_{v}(u, v)$ are strictly increasing functions about $u$ and $v$ for all $(u, v) \in \mathbb{R}^{2}$.

If $Y$ is a closed set of a topological space $X$, we denote by $\operatorname{cat}_{X}(Y)$ the Ljusternik-Schnirelmann category of $Y$ in $X$, namely the least number of closed and contractible sets in $X$ which cover $Y$. The main result we get is the following: 
Theorem 1.1. Assume that $\left(F_{0}\right)-\left(F_{2}\right)$ hold. In addition, suppose either $N \geq 8$ and $2<q<2^{*}$ or $N \geq 5$ and $q=2$. Then there exists $\Lambda>0$ such that the problem (1.1) has at least cat $\Omega(\Omega)$ distinct nontrivial solutions for $\lambda, \delta \in(0, \Lambda)$.

This paper is organized as follows. In Section 2, we show that some notations and Palais-Smale condition are established. We present some technical lemmas which are crucial in the proof of the main result in Section 3. Theorem 1.1 is proved in Section 4.

\section{Preliminaries and Palais-Smale condition}

Notations. Throughout this paper, we make use of the following notations.

- $C, C_{i}$ will denote various positive constants which can change from line to line.

- $\rightarrow$ (respectively $\rightarrow$ ) denotes strong (respectively weak) convergence.

- $O\left(\varepsilon^{t}\right)$ denote $\left|O\left(\varepsilon^{t}\right)\right| / \varepsilon^{t} \leq C, o_{m}(1)$ denote $o_{m}(1) \rightarrow 0$ as $m \rightarrow \infty$.

- $L^{s}(\Omega)(1 \leq s<+\infty)$ denote Lebesgue spaces, the norm $L^{s}$ is denoted by $|\cdot|_{s}$ for $1 \leq s<+\infty$.

- $B_{r}(x)$ denote a ball centered at $x$ with radius $r$.

- The dual space of a Banach space $E$ will be denoted by $E^{-1}$.

- The product space $E:=H_{0}^{2}(\Omega) \times H_{0}^{2}(\Omega)$ endowed with the norm $\|(u, v)\|_{E}$ $=\left(\|u\|_{H_{0}^{2}(\Omega)}^{2}+\|v\|_{H_{0}^{2}(\Omega)}^{2}\right)^{\frac{1}{2}}$, and the norm $\|u\|_{H_{0}^{2}(\Omega)}=\left(\int_{\Omega}|\Delta u|^{2} \mathrm{~d} x\right)^{\frac{1}{2}}$.

- $S$ is the best Sobolev constant defined by

$$
S=\inf _{u \in H_{0}^{2}(\Omega) \backslash\{0\}} \frac{\int_{\Omega}|\Delta u|^{2} d x}{\left(\int_{\Omega}|u|^{2^{*}} d x\right)^{\frac{2}{2^{*}}}} .
$$

From [18], we know that $S$ is achieved when $\Omega=\mathbb{R}^{N}$ by function

$$
U_{\varepsilon}(x)=\left(N(N-4)\left(N^{2}-4\right) \varepsilon^{2}\right)^{\frac{N-4}{8}}\left(\frac{1}{\varepsilon+|x|^{2}}\right)^{\frac{N-4}{2}}
$$

for all $\varepsilon>0$. Moreover, the function $U_{\varepsilon}(x)$ solves the equation $\Delta^{2} u=|u|^{2^{*}-2} u$ in $\mathbb{R}^{N}$ with $N \geq 5$ and

$$
\left|\Delta U_{\varepsilon}(x)\right|_{2}^{2}=\left|U_{\varepsilon}(x)\right|_{2^{*}}^{2^{*}}=S^{\frac{N}{4}}
$$

Now, we point out some important properties of homogeneous functions.

Let $\alpha \geq 1$ and $H$ be a differentiable $\alpha$-homogeneous function, then

(i) for all $s, t \in \mathbb{R}, s H_{s}(s, t)+t H_{t}(s, t)=\alpha H(s, t)$;

(ii) there exists $M_{H}>0$ such that $|H(s, t)| \leq M_{H}\left(|s|^{\alpha}+|t|^{\alpha}\right)$ for all $s, t \in \mathbb{R}$, where $M_{H}=\max \left\{H(s, t): s, t \in \mathbb{R},|s|^{\alpha}+|t|^{\alpha}=1\right\}$;

(iii) the maximum $M_{H}$ is attained for some $\left(s_{0}, t_{0}\right) \in \mathbb{R}^{2}$, i.e., $\left|s_{0}\right|^{\alpha}+\left|t_{0}\right|^{\alpha}=1$ and $H\left(s_{0}, t_{0}\right)=M_{H}$

(iv) $H_{s}(s, t), H_{t}(s, t)$ are $(\alpha-1)$ homogeneous.

By $\left(F_{0}\right)$ and the properties of homogeneous functions, we have

$$
F_{u}(u, v) u+F_{v}(u, v) v=2^{*} F(u, v)
$$


and

$$
F(u, v) \leq\left(M_{F}\left(|u|^{2}+|v|^{2}\right)\right)^{\frac{2^{*}}{2}}
$$

where

$$
M_{F}=\max \left\{(F(u, v))^{\frac{2}{2^{*}}}:(u, v) \in \mathbb{R}^{2},|u|^{2}+|v|^{2}=1\right\} .
$$

A pair of functions $(u, v) \in E$ is said to be a weak solution of problem (1.1) if

$$
\begin{aligned}
& \int_{\Omega} \Delta u \Delta \varphi_{1}+\Delta v \Delta \varphi_{2} d x-\int_{\Omega} F_{u}(u, v) \varphi_{1} d x-\int_{\Omega} F_{v}(u, v) \varphi_{2} d x \\
& -\lambda \int_{\Omega}|u|^{q-2} u \varphi_{1} d x-\delta \int_{\Omega}|v|^{q-2} v \varphi_{2} d x=0, \forall\left(\varphi_{1}, \varphi_{2}\right) \in E .
\end{aligned}
$$

The corresponding energy functional of problem (1.1) is defined on $E$ by

$\mathcal{E}_{\lambda, \delta}(u, v)=\frac{1}{2} \int_{\Omega}|\Delta u|^{2}+|\Delta v|^{2} d x-\int_{\Omega} F(u, v) d x-\frac{\lambda}{q} \int_{\Omega}|u|^{q} d x-\frac{\delta}{q} \int_{\Omega}|v|^{q} d x$.

Using assumptions $\left(F_{0}\right)-\left(F_{2}\right)$, we can verify $\mathcal{E}_{\lambda, \delta}(u, v) \in \mathcal{C}^{1}(E, \mathbb{R})$ (the proof is almost the same as that in [20]). It is well known that the weak solutions of problem (1.1) are the critical points of the energy functional $\mathcal{E}_{\lambda, \delta}$.

As the energy functional $\mathcal{E}_{\lambda, \delta}$ is not bounded below on $E$, we need to study $\mathcal{E}_{\lambda, \delta}$ on the Nehari manifold

$$
\mathcal{N}_{\lambda, \delta}=\left\{(u, v) \in E \backslash\{(0,0)\}:\left\langle\mathcal{E}_{\lambda, \delta}^{\prime}(u, v),(u, v)\right\rangle=0\right\},
$$

where $\mathcal{E}_{\lambda, \delta}^{\prime}(u, v)$ denotes the Fréchet derivative of $\mathcal{E}_{\lambda, \delta}$ at $(u, v)$, and $\langle\cdot, \cdot\rangle$ is the duality product between $E$ and its dual space $E^{-1}$. A direct computation shows that for all $(u, v) \in E \backslash\{(0,0)\}$, there exists a unique $t^{*}>0$ such that $t^{*}(u, v) \in \mathcal{N}_{\lambda, \delta}$. The maximum of the function $t \mapsto \mathcal{E}_{\lambda, \delta}(t(u, v))$, for $t \geq 0$, is achieved at $t=t^{*}$ (see Lemma 4.1 in [22]). Note that $\mathcal{N}_{\lambda, \delta}$ contains every nonzero solution of problem (1.1), and define the minimax $c_{\lambda, \delta}$ as

$$
c_{\lambda, \delta}=\inf _{(u, v) \in \mathcal{N}_{\lambda, \delta}} \mathcal{E}_{\lambda, \delta}(u, v)
$$

Moreover, we note that there exists $\rho>0$, such that

$$
\|(u, v)\|_{E} \geq \rho>0, \forall(u, v) \in \mathcal{N}_{\lambda, \delta} .
$$

It is standard to check that $\mathcal{E}_{\lambda, \delta}$ satisfies Mountain Pass geometry, so we can use the homogeneity of $F$ to prove that $c_{\lambda, \delta}$ can be alternatively characterized by

$$
c_{\lambda, \delta}=\inf _{\gamma \in \Gamma} \max _{t \in[0,1]} \mathcal{E}_{\lambda, \delta}(\gamma(t))=\inf _{(u, v) \in E \backslash\{(0,0)\}} \max _{t \geq 0} \mathcal{E}_{\lambda, \delta}(t(u, v))>0,
$$

where $\Gamma=\left\{\gamma \in C([0,1], E): \gamma(0)=0, \mathcal{E}_{\lambda, \delta}(\gamma(1))<0\right\}$. Its proofs can be done as Theorem 4.2 in [22]. 
In this section, we will find the range of $c$ where the $(P S)_{c}$ condition holds for the functional $\mathcal{E}_{\lambda, \delta}$. First let us define

$$
S_{F}:=\inf _{u, v \in H_{0}^{2}(\Omega) \backslash\{0\}}\left\{\frac{\int_{\Omega}|\Delta u|^{2}+|\Delta v|^{2} d x}{\left(\int_{\Omega} F(u, v) d x\right)^{\frac{2}{2^{*}}}}: \int_{\Omega} F(u, v) d x>0\right\} .
$$

Lemma 2.1. If $N \geq 5$ and $F$ satisfies $\left(F_{0}\right)-\left(F_{2}\right)$, then the functional $\mathcal{E}_{\lambda, \delta}$ satisfies the $(P S)_{c}$ condition for all $c<\frac{4}{N-4}\left(\frac{S_{F}}{2^{*}}\right)^{\frac{N}{4}}$, provide one of the following conditions holds

(i) $2<q<2^{*}$ and $\lambda, \delta>0$;

(ii) $q=2$, and $\lambda, \delta \in\left(0, \Lambda_{1}\right)$, where $\Lambda_{1}>0$ denotes the first eigenvalue of $\left(\Delta^{2}, H_{0}^{2}(\Omega)\right)$.

Proof. Let $\left\{\left(u_{m}, v_{m}\right)\right\} \subset E$ such that $\mathcal{E}_{\lambda, \delta}^{\prime}\left(u_{m}, v_{m}\right) \rightarrow 0$ and $\mathcal{E}_{\lambda, \delta}\left(u_{m}, v_{m}\right) \rightarrow$ $c<\frac{4}{N-4}\left(\frac{S_{F}}{2^{*}}\right)^{\frac{N}{4}}$. Now, we firstly prove that $\left\{\left(u_{m}, v_{m}\right)\right\}$ is bounded in $E$. If the above item (i) is true it suffices to use the definition of $I_{\lambda, \delta}$ to obtain $C_{1}>0$ such that

$$
\begin{aligned}
& c+C_{1}\left\|\left(u_{m}, v_{m}\right)\right\|_{E}+o_{m}(1) \\
\geq & \mathcal{E}_{\lambda, \delta}\left(u_{m}, v_{m}\right)-\frac{1}{q}\left\langle\mathcal{E}_{\lambda, \delta}^{\prime}\left(u_{m}, v_{m}\right),\left(u_{m}, v_{m}\right)\right\rangle \\
= & \left(\frac{1}{2}-\frac{1}{q}\right)\left\|\left(u_{m}, v_{m}\right)\right\|_{E}^{2}+\left(\frac{2^{*}}{q}-1\right) \int_{\Omega} F\left(u_{m}, v_{m}\right) d x \\
\geq & \frac{q-2}{2 q}\left\|\left(u_{m}, v_{m}\right)\right\|_{E}^{2} .
\end{aligned}
$$

The above expression implies that $\left\{\left(u_{m}, v_{m}\right)\right\} \subset E$ is bounded. In the case (ii), it follows that

$$
\begin{aligned}
\int_{\Omega}\left(\lambda\left|u_{m}\right|^{2}+\delta\left|v_{m}\right|^{2}\right) d x & \leq \max \{\lambda, \delta\} \int_{\Omega}\left(\left|u_{m}\right|^{2}+\left|v_{m}\right|^{2}\right) d x \\
& \leq \frac{\max \{\lambda, \delta\}}{\Lambda_{1}}\left\|\left(u_{m}, v_{m}\right)\right\|_{E}^{2}
\end{aligned}
$$

and therefore we get

$$
\begin{aligned}
& c+C_{1}\left\|\left(u_{m}, v_{m}\right)\right\|_{E}+o_{m}(1) \\
\geq & \mathcal{E}_{\lambda, \delta}\left(u_{m}, v_{m}\right)-\frac{1}{2^{*}}\left\langle\mathcal{E}_{\lambda, \delta}^{\prime}\left(u_{m}, v_{m}\right),\left(u_{m}, v_{m}\right)\right\rangle \\
= & \left(\frac{1}{2}-\frac{1}{2^{*}}\right)\left\|\left(u_{m}, v_{m}\right)\right\|_{E}^{2}+\left(\frac{1}{2^{*}}-\frac{1}{2}\right) \int_{\Omega}\left(\lambda\left|u_{m}\right|^{2}+\delta\left|v_{m}\right|^{2}\right) d x \\
\geq & \frac{2}{N}\left(1-\frac{\max \{\lambda, \delta\}}{\Lambda_{1}}\right)\left\|\left(u_{m}, v_{m}\right)\right\|_{E}^{2} .
\end{aligned}
$$

Since $\lambda, \delta \in\left(0, \Lambda_{1}\right)$, the boundedness of $\left\{\left(u_{m}, v_{m}\right)\right\}$ follows as the first case. 
So, $\left\{\left(u_{m}, v_{m}\right)\right\}$ is bounded in $E$. Going if necessary to a subsequence, we can assume that

$$
\begin{cases}\left(u_{m}, v_{m}\right) \rightarrow(u, v), & \text { in } E \\ \left(u_{m}, v_{m}\right) \rightarrow(u, v), & \text { a.e. in } \Omega, \\ \left(u_{m}, v_{m}\right) \rightarrow(u, v), & \text { in } L^{s}(\Omega) \times L^{s}(\Omega), 1 \leq s<2^{*},\end{cases}
$$

as $m \rightarrow \infty$. Clearly, we have

$$
\int_{\Omega}\left(\lambda\left|u_{m}\right|^{q}+\delta\left|v_{m}\right|^{q}\right) d x=\int_{\Omega}\left(\lambda|u|^{q}+\delta|v|^{q}\right) d x+o_{m}(1) .
$$

Moreover, a standard argument shows that $\mathcal{E}_{\lambda, \delta}^{\prime}(u, v)=0$. Thus we get

$$
\begin{aligned}
\mathcal{E}_{\lambda, \delta}(u, v) & =\frac{1}{2}\|(u, v)\|_{E}^{2}-\int_{\Omega} F(u, v) d x-\frac{1}{q} \int_{\Omega}\left(\lambda|u|^{q}+\delta|v|^{q}\right) d x \\
& =\left(\frac{1}{2}-\frac{1}{q}\right)\|(u, v)\|_{E}^{2}+\left(\frac{2^{*}}{q}-1\right) \int_{\Omega} F(u, v) d x \\
& \geq 0
\end{aligned}
$$
have

$$
\left\|\left(u_{m}, v_{m}\right)\right\|_{E}^{2}=\|(u, v)\|_{E}^{2}+\left\|\left(\tilde{u}_{m}, \tilde{v}_{m}\right)\right\|_{E}^{2}+o_{m}(1) .
$$

By the same method of Lemma 8 in [8] (or Lemma 3.4 in [20]), we obtain

$$
\int_{\Omega} F\left(u_{m}, v_{m}\right) d x=\int_{\Omega} F(u, v) d x+\int_{\Omega} F\left(\tilde{u}_{m}, \tilde{v}_{m}\right) d x+o_{m}(1) .
$$

By (2.10)-(2.13) and the weak convergence of $\left(u_{m}, v_{m}\right)$, we have

$$
\begin{aligned}
c+o_{m}(1) & =\mathcal{E}_{\lambda, \delta}(u, v)+\frac{1}{2}\left\|\left(\tilde{u}_{m}, \tilde{v}_{m}\right)\right\|_{E}^{2}-\int_{\Omega} F\left(\tilde{u}_{m}, \tilde{v}_{m}\right) d x \\
& \geq \frac{1}{2}\left\|\left(\tilde{u}_{m}, \tilde{v}_{m}\right)\right\|_{E}^{2}-\int_{\Omega} F\left(\tilde{u}_{m}, \tilde{v}_{m}\right) d x .
\end{aligned}
$$

By using $\mathcal{E}_{\lambda, \delta}^{\prime}\left(u_{m}, v_{m}\right) \rightarrow 0$ and $(2.10),(2.12)-(2.13)$, we get

$$
\begin{aligned}
o_{m}(1) & =\left\langle\mathcal{E}_{\lambda, \delta}^{\prime}\left(u_{m}, v_{m}\right),\left(u_{m}, v_{m}\right)\right\rangle \\
& =\left\|\left(u_{m}, v_{m}\right)\right\|_{E}^{2}-2^{*} \int_{\Omega} F\left(u_{m}, v_{m}\right) d x-\int_{\Omega}\left(\lambda\left|u_{m}\right|^{q}+\delta\left|v_{m}\right|^{q}\right) d x \\
& =\left\langle\mathcal{E}_{\lambda, \delta}^{\prime}(u, v),(u, v)\right\rangle+\left\|\left(\tilde{u}_{m}, \tilde{v}_{m}\right)\right\|_{E}^{2}-2^{*} \int_{\Omega} F\left(\tilde{u}_{m}, \tilde{v}_{m}\right) d x .
\end{aligned}
$$

Recalling that $\mathcal{E}_{\lambda, \delta}^{\prime}(u, v)=0$, we can use the above equality to obtain

$$
\lim _{m \rightarrow \infty}\left\|\left(\tilde{u}_{m}, \tilde{v}_{m}\right)\right\|_{E}^{2}=k=2^{*} \lim _{m \rightarrow \infty} \int_{\Omega} F\left(\tilde{u}_{m}, \tilde{v}_{m}\right) d x,
$$

where $k \geq 0$. 
In view of the definition of $S_{F}$, we have that

$$
\left\|\left(\tilde{u}_{m}, \tilde{v}_{m}\right)\right\|_{E}^{2} \geq S_{F}\left(\int_{\Omega} F\left(\tilde{u}_{m}, \tilde{v}_{m}\right) d x\right)^{\frac{2}{2^{*}}} .
$$

Taking the limit we get $k \geq S_{F}\left(\frac{k}{2^{*}}\right)^{\frac{2}{2^{*}}}$. So, if $k>0$, we conclude that $k \geq$ $2^{*}\left(\frac{S_{F}}{2^{*}}\right)^{\frac{N}{4}}$, therefore from $(2.14)$ and $(2.15)$, we get

$$
c \geq\left(\frac{1}{2}-\frac{1}{2^{*}}\right) k \geq \frac{4}{N-4}\left(\frac{S_{F}}{2^{*}}\right)^{\frac{N}{4}},
$$

which contradicts $c<\frac{4}{N-4}\left(\frac{S_{F}}{2^{*}}\right)^{\frac{N}{4}}$. Hence $k=0$ and therefore $\left(u_{m}, v_{m}\right) \rightarrow$ $(u, v)$ strongly in $E$. have

Before presenting our next result we remark that, using Lemma 3 in [8] we

$$
S_{F}=\frac{1}{M_{F}} S,
$$

where $S$ is the best constant defined in (2.1), $M_{F}$ defined in (2.6).

We define a cut-off function $\phi(x) \in \mathcal{C}_{0}^{\infty}\left(\mathbb{R}^{N}\right)$ such that $\phi(x)=1$ if $|x| \leq R$; $\phi(x)=0$ if $|x| \geq 2 R$ and $0 \leq \phi(x) \leq 1$, where $B_{2 R}(0) \subset \Omega$, set $u_{\varepsilon}=\frac{\phi(x) U_{\varepsilon}}{\left|\phi U_{\varepsilon}\right|_{2^{*}}}$, where $U_{\varepsilon}$ was defined in (2.2). So that $\left|u_{\varepsilon}\right|_{2^{*}}=1$. Then we can get the following results from Lemma 7.3 in [3]:

$$
\begin{gathered}
\left\|u_{\varepsilon}\right\|_{H_{0}^{2}(\Omega)}^{2}=S+O\left(\varepsilon^{N-4}\right), \\
\int_{\Omega}\left|u_{\varepsilon}\right|^{\xi} d x \approx \begin{cases}\varepsilon^{\frac{N-4}{2} \xi}, & \text { if } \xi<\frac{N}{N-4} \\
\varepsilon^{\frac{2 N-(N-4) \xi}{2}}|\ln \varepsilon|, & \text { if } \xi=\frac{N}{N-4} \\
\varepsilon^{\frac{2 N-(N-4) \xi}{2}}, & \text { if } \xi>\frac{N}{N-4},\end{cases}
\end{gathered}
$$

where $A \approx B$ means $C_{1} B \leq A \leq C_{2} B$.

Lemma 2.2. Suppose that $\left(F_{0}\right)-\left(F_{2}\right)$ hold, $N \geq 8,2<q<2^{*}$ and $\lambda>0, \delta>0$, then $c_{\lambda, \delta}<\frac{4}{N-4}\left(\frac{S_{F}}{2^{*}}\right)^{\frac{N}{4}}$. The same result holds if $N \geq 5, q=2$ and $\lambda, \delta \in$ $\left(0, \Lambda_{1}\right)$, where $\Lambda_{1}>0$ denotes the first eigenvalue of $\left(\Delta^{2}, H_{0}^{2}(\Omega)\right)$.

Proof. From the property (iii) of homogeneous functions, there exists $\left(e_{1}, e_{2}\right) \in$ $\mathbb{R}^{2}$ such that

$$
e_{1}^{2}+e_{2}^{2}=1 \text { and } F\left(e_{1}, e_{2}\right)=M_{F}^{\frac{2^{*}}{2}} .
$$

We can use the homogeneity of $F$ to get, for any $t \geq 0$,

$$
h(t):=\mathcal{E}_{\lambda, \delta}\left(t e_{1} u_{\varepsilon}, t e_{2} u_{\varepsilon}\right)=\frac{t^{2}}{2}\left\|u_{\varepsilon}\right\|_{H_{0}^{2}(\Omega)}^{2}-t^{2^{*}} F\left(e_{1}, e_{2}\right)-\frac{t^{q}}{q}\left(\lambda e_{1}^{q}+\delta e_{2}^{q}\right)\left|u_{\varepsilon}\right|_{q}^{q} .
$$

We shall consider two distinct cases.

Case 1. $N \geq 8,2<q<2^{*}$. 
Note that $\lim _{t \rightarrow+\infty} h(t)=-\infty, h(0)=0, h(t)>0$ for $t \rightarrow 0^{+}$. So $\sup _{t \geq 0} h(t)$ is attained at some $t_{\varepsilon}>0$ such that

$$
h\left(t_{\varepsilon}\right)=\max _{t \geq 0} h(t) .
$$

Let

$$
g(t)=\frac{t^{2}}{2}\left\|u_{\varepsilon}\right\|_{H_{0}^{2}(\Omega)}^{2}-t^{2^{*}} F\left(e_{1}, e_{2}\right), t \geq 0,
$$

and notice that the maximum value of $g(t)$ occurs at the point

$$
t_{\varepsilon}=\left(\frac{\left\|u_{\varepsilon}\right\|_{H_{0}^{2}(\Omega)}^{2}}{2^{*} F\left(e_{1}, e_{2}\right)}\right)^{\frac{1}{2^{*}-2}} .
$$

So, for each $t \geq 0$,

$$
g(t) \leq g\left(t_{\varepsilon}\right)=\frac{2}{N}\left(\frac{\left\|u_{\varepsilon}\right\|_{H_{0}^{2}(\Omega)}^{2}}{\left(2^{*} F\left(e_{1}, e_{2}\right)\right)^{\frac{2}{2^{*}}}}\right)^{\frac{N}{4}},
$$

and therefore

$$
h\left(t_{\varepsilon}\right) \leq \frac{2}{N}\left(\frac{\left\|u_{\varepsilon}\right\|_{H_{0}^{2}(\Omega)}^{2}}{\left(2^{*} F\left(e_{1}, e_{2}\right)\right)^{\frac{2}{2^{*}}}}\right)^{\frac{N}{4}}-\frac{t_{\varepsilon}^{q}}{q}\left(\lambda e_{1}^{q}+\delta e_{2}^{q}\right)\left|u_{\varepsilon}\right|_{q}^{q} .
$$

We claim that, for some $C_{2}>0$, there holds

$$
t_{\varepsilon}^{q}\left(\lambda e_{1}^{q}+\delta e_{2}^{q}\right) \geq C_{2} .
$$

Indeed, if this is not the case, we have that $t_{\varepsilon_{m}} \rightarrow 0$ for some sequence $\varepsilon_{m} \rightarrow 0^{+}$, then,

$$
0<c_{\lambda, \delta} \leq \sup _{t \geq 0} \mathcal{E}_{\lambda, \delta}\left(t e_{1} u_{\varepsilon_{m}}, t e_{2} u_{\varepsilon_{m}}\right)=\mathcal{E}_{\lambda, \delta}\left(t_{\varepsilon_{m}} e_{1} u_{\varepsilon_{m}}, t_{\varepsilon_{m}} e_{2} u_{\varepsilon_{m}}\right) \rightarrow 0
$$

which is a contradiction. So, the claim holds and we infer from (2.21) and (2.16)-(2.18) that

$$
\begin{aligned}
h\left(t_{\varepsilon}\right) & \leq \frac{2}{N}\left(\frac{S+O\left(\varepsilon^{N-4}\right)}{\left(2^{*} F\left(e_{1}, e_{2}\right)\right)^{\frac{2}{2^{*}}}}\right)^{\frac{N}{4}}-C_{3}\left|u_{\varepsilon}\right|_{q}^{q} \\
& =\frac{2}{N} 2^{*}\left(\frac{S+O\left(\varepsilon^{N-4}\right)}{2^{*} M_{F}}\right)^{\frac{N}{4}}-C_{3}\left|u_{\varepsilon}\right|_{q}^{q} \\
& \leq \frac{4}{N-4}\left(\frac{S_{F}}{2^{*}}\right)^{\frac{N}{4}}+O\left(\varepsilon^{N-4}\right)-C_{3}\left|u_{\varepsilon}\right|_{q}^{q} \\
& \leq \frac{4}{N-4}\left(\frac{S_{F}}{2^{*}}\right)^{\frac{N}{4}}+O\left(\varepsilon^{N-4}\right)-O\left(\varepsilon^{\frac{2 N-(N-4) q}{2}}\right),
\end{aligned}
$$

where $C_{3}=\frac{C_{2}}{q}$. By $N \geq 8$, we obtain $N-4>\frac{2 N-(N-4) q}{2}$. Thus, from the above inequality we conclude that, for each $\varepsilon>0$ small, there holds

$$
c_{\lambda, \delta} \leq \sup _{t \geq 0} \mathcal{E}_{\lambda, \delta}\left(t e_{1} u_{\varepsilon}, t e_{2} u_{\varepsilon}\right)=h\left(t_{\varepsilon}\right)<\frac{4}{N-4}\left(\frac{S_{F}}{2^{*}}\right)^{\frac{N}{4}} .
$$

Case 2. $N \geq 5, q=2$. 
In this case we have that $h^{\prime}(t)=0$ if and only if,

$$
\left\|u_{\varepsilon}\right\|_{H_{0}^{2}(\Omega)}^{2}-\left(\lambda e_{1}^{2}+\delta e_{2}^{2}\right)\left|u_{\varepsilon}\right|_{2}^{2}=2^{*} t^{2^{*}-2} F\left(e_{1}, e_{2}\right) .
$$

Since we suppose $\lambda, \delta \in\left(0, \Lambda_{1}\right)$, by Poincaré's inequality and (2.19), we obtain

$$
\begin{aligned}
\left(\lambda e_{1}^{2}+\delta e_{2}^{2}\right)\left|u_{\varepsilon}\right|_{2}^{2} & \leq \max \{\lambda, \delta\}\left(e_{1}^{2}+e_{2}^{2}\right)\left|u_{\varepsilon}\right|_{2}^{2} \\
& <\Lambda_{1}\left|u_{\varepsilon}\right|_{2}^{2} \leq\left\|u_{\varepsilon}\right\|_{H_{0}^{2}(\Omega)}^{2} .
\end{aligned}
$$

Thus, there exists $t_{\varepsilon}>0$ satisfying (2.20).

Arguing as the first case, from (2.22) and Lemma 2 in [11], we have

$$
\begin{aligned}
h\left(t_{\varepsilon}\right) & \leq \frac{4}{N-4}\left(\frac{S_{F}}{2^{*}}\right)^{\frac{N}{4}}+O\left(\varepsilon^{N-4}\right)-C_{3}\left|u_{\varepsilon}\right|_{2}^{2} \\
& =\frac{4}{N-4}\left(\frac{S_{F}}{2^{*}}\right)^{\frac{N}{4}}+O\left(\varepsilon^{N-4}\right)- \begin{cases}C \varepsilon^{4}+O\left(\varepsilon^{N-4}\right), & N>8, \\
C \varepsilon^{4}|\ln \varepsilon|+O\left(\varepsilon^{4}\right), & N=8, \\
C \varepsilon^{N-4}+O\left(\varepsilon^{4}\right), & N=5,6,7 .\end{cases}
\end{aligned}
$$

Choosing $\varepsilon>0$ small enough, we have

$$
c_{\lambda, \delta} \leq \sup _{t \geq 0} \mathcal{E}_{\lambda, \delta}\left(t e_{1} u_{\varepsilon}, t e_{2} u_{\varepsilon}\right)=h\left(t_{\varepsilon}\right)<\frac{4}{N-4}\left(\frac{S_{F}}{2^{*}}\right)^{\frac{N}{4}} .
$$

This concludes the proof.

By Lemmas 2.1 and 2.2, we can obtain the following result.

Theorem 2.3. Suppose that $\left(F_{0}\right)-\left(F_{2}\right)$ hold, then the problem (1.1) has at least one nontrivial solution for $N \geq 8,2<q<2^{*}$ and $\lambda, \delta>0$, or $N \geq 5, q=2$ and $\lambda, \delta \in\left(0, \Lambda_{1}\right)$, where $\Lambda_{1}$ is the first eigenvalue of $\left(\Delta^{2}, H_{0}^{2}(\Omega)\right)$.

Proof. Since $\mathcal{E}_{\lambda, \delta}$ satisfies the geometric conditions of the Mountain Pass Theorem, there exists $\left\{\left(u_{m}, v_{m}\right)\right\} \subset E$ such that $\mathcal{E}_{\lambda, \delta}\left(u_{m}, v_{m}\right) \rightarrow c_{\lambda, \delta}, \mathcal{E}_{\lambda, \delta}^{\prime}\left(u_{m}, v_{m}\right) \rightarrow$ 0 . It follows from Lemmas 2.1 and 2.2 that $\left\{\left(u_{m}, v_{m}\right)\right\}$ converges, along a subsequence, to a nonzero critical point $(u, v) \in E$ of $\mathcal{E}_{\lambda, \delta}$. Theorem 2.3 is proved.

We finalize this section with the study of the asymptotic behavior of the minimax level $c_{\lambda, \delta}$ as both the parameters $\lambda, \delta$ approach zero.

Lemma 2.4. $\lim _{\lambda, \delta \rightarrow 0^{+}} c_{\lambda, \delta}=c_{0,0}=\frac{4}{N-4}\left(\frac{S_{F}}{2^{*}}\right)^{\frac{N}{4}}$.

Proof. We first prove the second equality. It follows from $\lambda=\delta=0$ that $\lambda|u|^{q}+\delta|v|^{q} \equiv 0$. If $e_{1}, e_{2}, u_{\varepsilon}$ and $t_{\varepsilon}$ are the same as those in the proof of Lemma 2.2, we have that $\left(t_{\varepsilon} e_{1} u_{\varepsilon}, t_{\varepsilon} e_{2} u_{\varepsilon}\right) \in \mathcal{N}_{0,0}$. Thus

$$
\begin{aligned}
c_{0,0} & \leq \mathcal{E}_{0,0}\left(t_{\varepsilon} e_{1} u_{\varepsilon}, t_{\varepsilon} e_{2} u_{\varepsilon}\right) \\
& =\frac{2}{N}\left(\frac{\left(e_{1}^{2}+e_{2}^{2}\right)\left\|u_{\varepsilon}\right\|_{H_{0}^{2}(\Omega)}^{2}}{\left(2^{*} F\left(e_{1}, e_{2}\right)\right)^{\frac{2}{2^{*}}}}\right)^{\frac{N}{4}} \\
& =\frac{2}{N}\left(\frac{S+O\left(\varepsilon^{N-4}\right)}{\left(2^{*} F\left(e_{1}, e_{2}\right)\right)^{\frac{2}{2^{*}}}}\right)^{\frac{N}{4}}
\end{aligned}
$$




$$
=\frac{4}{N-4}\left(\frac{S+O\left(\varepsilon^{N-4}\right)}{2^{*} M_{F}}\right)^{\frac{N}{4}} .
$$

Taking the limit as $\varepsilon \rightarrow 0^{+}$and using (2.16), we conclude that $c_{0,0} \leq \frac{4}{N-4}\left(\frac{S_{F}}{2^{*}}\right)^{\frac{N}{4}}$.

In order to obtain the reverse inequality we consider $\left\{\left(u_{m}, v_{m}\right)\right\} \subset E$ such that $\mathcal{E}_{0,0}\left(u_{m}, v_{m}\right) \rightarrow c_{0,0}$ and $\mathcal{E}_{0,0}^{\prime}\left(u_{m}, v_{m}\right) \rightarrow 0$. It is easy to show that the sequence $\left\{\left(u_{m}, v_{m}\right)\right\}$ is bounded in $E$ and therefore $\left\langle\mathcal{E}_{0,0}^{\prime}\left(u_{m}, v_{m}\right),\left(u_{m}, v_{m}\right)\right\rangle=$ $\left\|\left(u_{m}, v_{m}\right)\right\|_{E}^{2}-2^{*} \int_{\Omega} F\left(u_{m}, v_{m}\right) d x=o_{m}(1)$. It follows that

$$
\lim _{m \rightarrow \infty}\left\|\left(u_{m}, v_{m}\right)\right\|_{E}^{2}=l=2^{*} \lim _{m \rightarrow \infty} \int_{\Omega} F\left(u_{m}, v_{m}\right) d x .
$$

Taking the limit in the inequality $S_{F}\left(\int_{\Omega} F\left(u_{m}, v_{m}\right) d x\right)^{\frac{2}{2^{*}}} \leq\left\|\left(u_{m}, v_{m}\right)\right\|_{E}^{2}$, we conclude that $N c_{0,0}=l \geq 2^{*}\left(\frac{S_{F}}{2^{*}}\right)^{\frac{N}{4}}$. Hence,

$$
\begin{aligned}
c_{0,0}=\lim _{m \rightarrow \infty} \mathcal{E}_{0,0}\left(u_{m}, v_{m}\right) & =\lim _{m \rightarrow \infty}\left(\frac{1}{2}\left\|\left(u_{m}, v_{m}\right)\right\|_{E}^{2}-\int_{\Omega} F\left(u_{m}, v_{m}\right) d x\right) \\
& =\frac{2}{N} l \geq \frac{4}{N-4}\left(\frac{S_{F}}{2^{*}}\right)^{\frac{N}{4}}
\end{aligned}
$$

and therefore $c_{0,0}=\frac{4}{N-4}\left(\frac{S_{F}}{2^{*}}\right)^{\frac{N}{4}}$.

We proceed now with the calculation of $\lim _{\lambda, \delta \rightarrow 0^{+}} c_{\lambda, \delta}$. Let $\left\{\lambda_{m}\right\},\left\{\delta_{m}\right\} \subset$ $\mathbb{R}^{+}$such that $\lambda_{m}, \delta_{m} \rightarrow 0^{+}$. Since $\lambda_{m}, \delta_{m}$ are positive, we have that $\int_{\Omega}\left(\lambda_{m}|u|^{q}+\right.$ $\left.\delta_{m}|v|^{q}\right) d x \geq 0$ whenever $(u, v)$ is nonnegative. Thus, for this kind of function, we have that $\mathcal{E}_{\lambda_{m}, \delta_{m}}(u, v) \leq \mathcal{E}_{0,0}(u, v)$. Then we have that

$$
\begin{aligned}
c_{\lambda_{m}, \delta_{m}} & =\inf _{(u, v) \neq(0,0)} \max _{t \geq 0} \mathcal{E}_{\lambda_{m}, \delta_{m}}(t(u, v)) \\
& \leq \inf _{\substack{(u, v) \neq(0,0),(u, v) \geq 0}} \max _{t \geq 0} \mathcal{E}_{\lambda_{m}, \delta_{m}}(t(u, v)) \\
& \leq \inf _{\substack{(u, v) \neq(0,0),(u, v) \geq 0}} \max _{t \geq 0} \mathcal{E}_{0,0}(t(u, v))=c_{0,0},
\end{aligned}
$$

in the last equality, we have used the infimum $c_{0,0}$ which can be attained at a nonnegative solution. The above inequality implies that

$$
\limsup _{m \rightarrow \infty} c_{\lambda_{m}, \delta_{m}} \leq c_{0,0} .
$$

On the other hand, it follows from Theorem 2.3 that there exists $\left\{\left(u_{m}, v_{m}\right)\right\}$ $\subset E$ such that

$$
\mathcal{E}_{\lambda_{m}, \delta_{m}}\left(u_{m}, v_{m}\right)=c_{\lambda_{m}, \delta_{m}}, \quad \mathcal{E}_{\lambda_{m}, \delta_{m}}^{\prime}\left(u_{m}, v_{m}\right) \rightarrow 0 .
$$

Since $c_{\lambda_{m}, \delta_{m}}$ is bounded, the same argument performed in the proof of Lemma 2.1 implies that $\left\{\left(u_{m}, v_{m}\right)\right\}$ is bounded in $E$. Since

$$
\lim _{m \rightarrow \infty} \int_{\Omega}\left(\lambda_{m}\left|u_{m}\right|^{q}+\delta_{m}\left|v_{m}\right|^{q}\right) d x=0 .
$$


Let $t_{m}>0$ be such that $t_{m}\left(u_{m}, v_{m}\right) \in \mathcal{N}_{0,0}$. Since $\left(u_{m}, v_{m}\right) \in \mathcal{N}_{\lambda_{m}, \delta_{m}}$, we have that

$$
\begin{aligned}
c_{0,0} & \leq \mathcal{E}_{0,0}\left(t_{m}\left(u_{m}, v_{m}\right)\right) \\
& =\mathcal{E}_{\lambda_{m}, \delta_{m}}\left(t_{m}\left(u_{m}, v_{m}\right)\right)+\frac{t_{m}^{q}}{q} \int_{\Omega}\left(\lambda_{m}\left|u_{m}\right|^{q}+\delta_{m}\left|v_{m}\right|^{q}\right) d x \\
& \leq \mathcal{E}_{\lambda_{m}, \delta_{m}}\left(u_{m}, v_{m}\right)+\frac{t_{m}^{q}}{q} \int_{\Omega}\left(\lambda_{m}\left|u_{m}\right|^{q}+\delta_{m}\left|v_{m}\right|^{q}\right) d x \\
& =c_{\lambda_{m}, \delta_{m}}+\frac{t_{m}^{q}}{q} \int_{\Omega}\left(\lambda_{m}\left|u_{m}\right|^{q}+\delta_{m}\left|v_{m}\right|^{q}\right) d x .
\end{aligned}
$$

If $\left\{t_{m}\right\}$ is bounded, we can use the above estimate and (2.24) to get

$$
c_{0,0} \leq \liminf _{m \rightarrow \infty} c_{\lambda_{m}, \delta_{m}}
$$

This and (2.23) we get

$$
c_{0,0} \leq \liminf _{m \rightarrow \infty} c_{\lambda_{m}, \delta_{m}} \leq \limsup _{m \rightarrow \infty} c_{\lambda_{m}, \delta_{m}} \leq c_{0,0}
$$

that is $c_{0,0}=\lim _{m \rightarrow \infty} c_{\lambda_{m}, \delta_{m}}$.

It remains to check that $\left\{t_{m}\right\}$ is bounded. A straightforward calculation shows that

$$
t_{m}=\left(\frac{\left\|\left(u_{m}, v_{m}\right)\right\|_{E}^{2}}{2^{*} \int_{\Omega} F\left(u_{m}, v_{m}\right) d x}\right)^{\frac{1}{2^{*}-2}} .
$$

Since $\left(u_{m}, v_{m}\right) \in \mathcal{N}_{\lambda_{m}, \delta_{m}}$, we obtain

$$
\begin{aligned}
\left\|\left(u_{m}, v_{m}\right)\right\|_{E}^{2} & =2^{*} \int_{\Omega} F\left(u_{m}, v_{m}\right) d x+\int_{\Omega}\left(\lambda_{m}\left|u_{m}\right|^{q}+\delta_{m}\left|v_{m}\right|^{q}\right) d x \\
& \leq 2^{*} S_{F}^{-\frac{2^{*}}{2}}\left\|\left(u_{m}, v_{m}\right)\right\|_{E}^{2^{*}}+o_{m}(1) .
\end{aligned}
$$

Hence $\left\|\left(u_{m}, v_{m}\right)\right\|_{E}^{2} \geq C_{4}>0$, and therefore from the above expression it follows that $\int_{\Omega} F\left(u_{m}, v_{m}\right) d x \geq C_{5}>0$. Thus, the boundedness of $\left\{\left(u_{m}, v_{m}\right)\right\}$ and (2.25) imply that $\left\{t_{m}\right\}$ is bounded. This completes the proof.

\section{Some technical results}

The following lemma is standard, and its proof follows adapting arguments found in [22].

Lemma 3.1. Suppose $\left\{\left(u_{m}, v_{m}\right)\right\} \subset E$ such that $\int_{\Omega} F\left(u_{m}, v_{m}\right) d x=1$ and $\lim _{m \rightarrow \infty}\left\|\left(u_{m}, v_{m}\right)\right\|_{E}^{2}=S_{F}$. Then there exist $\left\{r_{m}\right\} \subset(0,+\infty)$ and $\left\{y_{m}\right\} \subset \mathbb{R}^{N}$ such that

$$
\omega_{m}(x)=\left(\omega_{m}^{1}(x), \omega_{m}^{2}(x)\right)=r_{m}^{\frac{N-4}{2}}\left(u_{m}\left(r_{m} x+y_{m}\right), v_{m}\left(r_{m} x+y_{m}\right)\right)
$$

contains a convergent subsequence denoted again by $\left\{\omega_{m}\right\}$ such that $\omega_{m} \rightarrow \omega$ in $\mathcal{D}^{2,2}\left(\mathbb{R}^{N}\right) \times \mathcal{D}^{2,2}\left(\mathbb{R}^{N}\right)$. Moreover, as $m \rightarrow \infty$, we have $r_{m} \rightarrow 0$ and $y_{m} \rightarrow y \in \bar{\Omega}$. 
Up to translations, we may assume that $0 \in \Omega$, since $\Omega$ is a smooth bounded domain of $\mathbb{R}^{N}$, we can choose $r>0$ small enough such that $B_{r}=B_{r}(0)=\{x \in$ $\left.\mathbb{R}^{N}: d(x, 0)<r\right\} \subset \Omega$ and the sets

$$
\Omega_{r}^{+}=\left\{x \in \mathbb{R}^{N}: \operatorname{dist}(x, \Omega)<r\right\}, \quad \Omega_{r}^{-}=\left\{x \in \mathbb{R}^{N}: \operatorname{dist}(x, \partial \Omega)>r\right\},
$$

are homotopically equivalent to $\Omega$. Let

$$
H_{0, \text { rad }}^{2}\left(B_{r}\right)=\left\{u \in H_{0}^{2}\left(B_{r}\right): u \text { is radial }\right\}
$$

and

We define the functional

$$
E_{\text {rad }}\left(B_{r}\right)=H_{0, \text { rad }}^{2}\left(B_{r}\right) \times H_{0, \text { rad }}^{2}\left(B_{r}\right) .
$$

$\mathcal{E}_{B_{r}}(u, v)=\frac{1}{2} \int_{B_{r}}\left(|\Delta u|^{2}+|\Delta v|^{2}\right) d x-\int_{B_{r}} F(u, v) d x-\frac{1}{q} \int_{B_{r}}\left(\lambda|u|^{q}+\delta|v|^{q}\right) d x$, $(u, v) \in E_{\text {rad }}\left(B_{r}\right)$, and set

$$
m_{\lambda, \delta}=\inf _{(u, v) \in \mathcal{N}_{\lambda, \delta}^{B r}} \mathcal{E}_{B_{r}}(u, v),
$$

where

$$
\mathcal{N}_{\lambda, \delta}^{B_{r}}:=\left\{(u, v) \in E_{\text {rad }}\left(B_{r}\right) \backslash\{(0,0)\}:\left\langle\mathcal{E}_{B_{r}}^{\prime}(u, v),(u, v)\right\rangle=0\right\} .
$$

Clearly, $m_{\lambda, \delta}$ is nonincreasing in $\lambda, \delta$. Note that $m_{\lambda, \delta}>0$ for all $\lambda, \delta>0$.

Arguing as in the proof of Lemma 2.4 and Theorem 2.3, we obtain the following result.

Lemma 3.2. Suppose that $\left(F_{0}\right)-\left(F_{2}\right)$ hold, then the infimum $m_{\lambda, \delta}$ is attained by a positive radial function $\left(u_{\lambda, \delta}, v_{\lambda, \delta}\right) \in E_{\text {rad }}$ whenever $N \geq 8,2<q<2^{*}$ and $\lambda, \delta>0$, or $N \geq 5, q=2$ and $\lambda, \delta \in\left(0, \Lambda_{1, \text { rad }}\right)$, where $\Lambda_{1, \text { rad }}>0$ is the first eigenvalue of the operator $\left(\Delta^{2}, H_{0, \text { rad }}^{2}\left(B_{r}\right)\right)$. Moreover,

$$
m_{\lambda, \delta}<\frac{4}{N-4}\left(\frac{S_{F}}{2^{*}}\right)^{\frac{N}{4}}, \quad \lim _{\lambda, \delta \rightarrow 0^{+}} m_{\lambda, \delta}=\frac{4}{N-4}\left(\frac{S_{F}}{2^{*}}\right)^{\frac{N}{4}} .
$$

We define the barycenter map $\beta: \mathcal{N}_{\lambda, \delta} \rightarrow \mathbb{R}^{N}$ by setting

$$
\beta(u, v)=\left(\frac{S_{F}}{2^{*}}\right)^{-\frac{N}{4}} \int_{\Omega} F(u, v) x d x .
$$

This map has the following property.

Lemma 3.3. If $N \geq 5,2 \leq q<2^{*}$ and $F$ satisfies $\left(F_{0}\right)-\left(F_{2}\right)$, then there exists $\lambda^{*}>0$ such that $\beta(u, v) \in \Omega_{r}^{+}$whenever $(u, v) \in \mathcal{N}_{\lambda, \delta}, \lambda, \delta \in\left(0, \lambda^{*}\right)$ and $\mathcal{E}_{\lambda, \delta}(u, v) \leq m_{\lambda, \delta}$.

Proof. We argue by contradiction. Suppose that there exist $\left\{\lambda_{m}\right\},\left\{\delta_{m}\right\} \subset \mathbb{R}^{+}$ and $\left\{\left(u_{m}, v_{m}\right)\right\} \subset \mathcal{N}_{\lambda_{m}, \delta_{m}}$ such that $\lambda_{m}, \delta_{m} \rightarrow 0^{+}$as $m \rightarrow \infty, \mathcal{E}_{\lambda_{m}, \delta_{m}}\left(u_{m}, v_{m}\right)$ $\leq m_{\lambda_{m}, \delta_{m}}$ but $\beta\left(u_{m}, v_{m}\right) \notin \Omega_{r}^{+}$. From $\left\{\left(u_{m}, v_{m}\right)\right\} \subset \mathcal{N}_{\lambda_{m}, \delta_{m}}$ and $\mathcal{E}_{\lambda_{m}, \delta_{m}}\left(u_{m}\right.$, $\left.v_{m}\right) \leq m_{\lambda_{m}, \delta_{m}}$, we have that $\left\{\left(u_{m}, v_{m}\right)\right\}$ is bounded in $E$. Moreover,

$$
0=\left\langle\mathcal{E}_{\lambda_{m}, \delta_{m}}^{\prime}\left(u_{m}, v_{m}\right),\left(u_{m}, v_{m}\right)\right\rangle
$$




$$
=\left\|\left(u_{m}, v_{m}\right)\right\|_{E}^{2}-2^{*} \int_{\Omega} F\left(u_{m}, v_{m}\right) d x-\int_{\Omega}\left(\lambda_{m}\left|u_{m}\right|^{q}+\delta_{m}\left|v_{m}\right|^{q}\right) d x .
$$

Since $\lambda_{m}, \delta_{m} \rightarrow 0^{+}$, we can use the boundedness of $\left\{\left(u_{m}, v_{m}\right)\right\}$ to get

$$
0 \leq \int_{\Omega}\left(\lambda_{m}\left|u_{m}\right|^{q}+\delta_{m}\left|v_{m}\right|^{q}\right) d x \rightarrow 0
$$

from which it follows that

$$
\lim _{m \rightarrow \infty}\left\|\left(u_{m}, v_{m}\right)\right\|_{E}^{2}=2^{*} \lim _{m \rightarrow \infty} \int_{\Omega} F\left(u_{m}, v_{m}\right) d x=k \geq 0 .
$$

Notice that

$$
\begin{aligned}
c_{\lambda_{m}, \delta_{m}} & \leq \mathcal{E}_{\lambda_{m}, \delta_{m}}\left(u_{m}, v_{m}\right) \\
& =\frac{1}{2}\left\|\left(u_{m}, v_{m}\right)\right\|_{E}^{2}-\int_{\Omega} F\left(u_{m}, v_{m}\right) d x-\frac{1}{q} \int_{\Omega}\left(\lambda_{m}\left|u_{m}\right|^{q}+\delta_{m}\left|v_{m}\right|^{q}\right) d x \\
& \leq m_{\lambda_{m}, \delta_{m}} .
\end{aligned}
$$

Recalling that $c_{\lambda_{m}, \delta_{m}}$ and $m_{\lambda_{m}, \delta_{m}}$ both converge to $\frac{4}{N-4}\left(\frac{S_{F}}{2^{*}}\right)^{\frac{N}{4}}$, we can use the above expression and $\int_{\Omega}\left(\lambda_{m}\left|u_{m}\right|^{q}+\delta_{m}\left|v_{m}\right|^{q}\right) d x \rightarrow 0$ again to conclude that $k=2^{*}\left(\frac{S_{F}}{2^{*}}\right)^{\frac{N}{4}}$, that is,

$$
\lim _{m \rightarrow \infty}\left\|\left(u_{m}, v_{m}\right)\right\|_{E}^{2}=2^{*}\left(\frac{S_{F}}{2^{*}}\right)^{\frac{N}{4}}=2^{*} \lim _{m \rightarrow \infty} \int_{\Omega} F\left(u_{m}, v_{m}\right) d x .
$$

Let $t_{m}=\left(\int_{\Omega} F\left(u_{m}, v_{m}\right) d x\right)^{-\frac{1}{2^{*}}}>0$ and notice that $t_{m}\left(u_{m}, v_{m}\right)$ satisfies the hypotheses of Lemma 3.1. Using Lemma 3.1, there exist sequences $\left\{r_{m}\right\} \subset$ $(0,+\infty)$ and $\left\{y_{m}\right\} \subset \mathbb{R}^{N}$ satisfying $r_{m} \rightarrow 0, y_{m} \rightarrow y \in \bar{\Omega}$ we have that $\omega_{m} \rightarrow \omega$ in $\mathcal{D}^{2,2}\left(\mathbb{R}^{N}\right) \times \mathcal{D}^{2,2}\left(\mathbb{R}^{N}\right)$.

Using the definition of $\beta,(3.2)$, the strong convergence of $\left\{\omega_{m}\right\}$ and Lebesgue's Theorem, we get

$$
\begin{aligned}
\beta\left(u_{m}, v_{m}\right) & =t_{m}^{-2^{*}}\left(\frac{S_{F}}{2^{*}}\right)^{-\frac{N}{4}} \int_{\Omega} F\left(t_{m}\left(u_{m}, v_{m}\right)\right) x d x \\
& =\left(1+o_{m}(1)\right) \int_{\Omega} F\left(t_{m}\left(u_{m}, v_{m}\right)\right) x d x \\
& =\left(1+o_{m}(1)\right) \int_{\Omega} F\left(\omega_{m}\right)\left(r_{m} x+y_{m}\right) d x \\
& =\left(1+o_{m}(1)\right)\left(\int_{\Omega} F(\omega) \bar{y} d x+o_{m}(1)\right) .
\end{aligned}
$$

Since $\bar{y} \in \bar{\Omega}$ and $\int_{\Omega} F(\omega) d x=1$, the above expression implies that

$$
\lim _{m \rightarrow \infty} \operatorname{dist}\left(\beta\left(u_{m}, v_{m}\right), \bar{\Omega}\right)=0,
$$

which contradicts $\beta\left(u_{m}, v_{m}\right) \notin \Omega_{r}^{+}$. 
According to Lemma 3.2, for each $\lambda, \delta>0$ small the infimum $m_{\lambda, \delta}$ is attained by a nonnegative radial function $\sigma_{\lambda, \delta}=\left(u_{\lambda, \delta}, v_{\lambda, \delta}\right) \in \mathcal{N}_{\lambda, \delta}^{B_{r}}$. We consider

$$
\mathcal{E}_{\lambda, \delta}^{m_{\lambda, \delta}}=\left\{(u, v) \in E: \mathcal{E}_{\lambda, \delta}(u, v) \leq m_{\lambda, \delta}\right\}
$$

and define the function $\gamma: \Omega_{r}^{-} \rightarrow \mathcal{E}_{\lambda, \delta}^{m_{\lambda, \delta}}$ by setting, for each $y \in \Omega_{r}^{-}$,

$$
\gamma(y)= \begin{cases}\sigma_{\lambda, \delta}(x-y), & \text { if } x \in B_{r}(y), \\ 0, & \text { otherwise. }\end{cases}
$$

A change of variables and straightforward calculations show that the map $\gamma$ is well defined. Since $\left(u_{\lambda, \delta}, v_{\lambda, \delta}\right)$ is radial, we have that $\int_{B_{r}} F\left(u_{\lambda, \delta}, v_{\lambda, \delta}\right) x d x=0$. Hence, for each $y \in \Omega_{r}^{-}$, we obtain

$$
\begin{aligned}
(\beta \circ \gamma)(y) & =\left(\frac{S_{F}}{2^{*}}\right)^{-\frac{N}{4}} \int_{\Omega} F\left(u_{\lambda, \delta}(x-y), v_{\lambda, \delta}(x-y)\right) x d x \\
& =\left(\frac{S_{F}}{2^{*}}\right)^{-\frac{N}{4}} \int_{\Omega} F\left(u_{\lambda, \delta}(t), v_{\lambda, \delta}(t)\right)(t+y) d t \\
& =\left(\frac{S_{F}}{2^{*}}\right)^{-\frac{N}{4}} \int_{\Omega} F\left(u_{\lambda, \delta}(t), v_{\lambda, \delta}(t)\right) y d t \\
& =y \alpha_{\lambda, \delta}
\end{aligned}
$$

where $\alpha_{\lambda, \delta}=\left(\frac{S_{F}}{2^{*}}\right)^{-\frac{N}{4}} \int_{\Omega} F\left(u_{\lambda, \delta}(t), v_{\lambda, \delta}(t)\right) d t$.

Along the way of proving Lemma 3.3, we have the following result.

Lemma 3.4. If $\lambda, \delta \rightarrow 0^{+}, \alpha_{\lambda, \delta} \rightarrow 1$.

Proof. By Lemma 3.2, we have that

$$
\begin{aligned}
m_{\lambda, \delta}= & \frac{1}{2} \int_{B_{r}}\left(\left|\Delta u_{\lambda, \delta}\right|^{2}+\left|\Delta v_{\lambda, \delta}\right|^{2}\right) d x-\int_{B_{r}} F\left(u_{\lambda, \delta}, v_{\lambda, \delta}\right) d x \\
& -\frac{1}{q} \int_{B_{r}}\left(\lambda\left|u_{\lambda, \delta}\right|^{q}+\delta\left|v_{\lambda, \delta}\right|^{q}\right) d x \\
< & \frac{4}{N-4}\left(\frac{S_{F}}{2^{*}}\right)^{\frac{N}{4}} .
\end{aligned}
$$

As before $\int_{B_{r}}\left(\lambda\left|u_{\lambda, \delta}\right|^{q}+\delta\left|v_{\lambda, \delta}\right|^{q}\right) d x \rightarrow 0$. Thus, $\mathcal{E}_{B_{r}}^{\prime}\left(u_{\lambda, \delta}, v_{\lambda, \delta}\right)=0$, the above expression and the same arguments used in the proof of Lemma 3.2 imply that

$$
\int_{\Omega} F\left(u_{\lambda, \delta}, v_{\lambda, \delta}\right) d x \rightarrow\left(\frac{S_{F}}{2^{*}}\right)^{\frac{N}{4}}
$$

The above equality and the definition of $\alpha_{\lambda, \delta}$ imply that $\alpha_{\lambda, \delta} \rightarrow 1$.

Next we define $H_{\lambda, \delta}:[0,1] \times\left(\mathcal{N}_{\lambda, \delta} \cap \mathcal{E}_{\lambda, \delta}^{m_{\lambda, \delta}}\right) \rightarrow \mathbb{R}^{N}$ by

$$
H_{\lambda, \delta}(t, z)=\left(t+\frac{1-t}{\alpha_{\lambda, \delta}}\right) \beta(z)
$$

We have the following: 
Lemma 3.5. If $F$ satisfies $\left(F_{0}\right)-\left(F_{2}\right)$, then there exists $\lambda^{* *}>0$ such that

$$
H_{\lambda, \delta}\left([0,1] \times\left(\mathcal{N}_{\lambda, \delta} \cap \mathcal{E}_{\lambda, \delta}^{m_{\lambda, \delta}}\right)\right) \subset \Omega_{r}^{+}
$$

for all $\lambda, \delta \in\left(0, \lambda^{* *}\right)$.

Proof. Arguing by contradiction, we suppose that there exist $t_{m} \in[0,1], \lambda_{m}$, $\delta_{m} \rightarrow 0^{+}$as $m \rightarrow \infty$, and $\left(u_{m}, v_{m}\right) \in \mathcal{N}_{\lambda, \delta} \cap \mathcal{E}_{\lambda, \delta}^{m_{\lambda, \delta}}$ such that $H_{\lambda_{m}, \delta_{m}}\left(t_{m}, u_{m}\right.$, $\left.v_{m}\right) \notin \Omega_{r}^{+}$for all $m$. Up to a subsequence $t_{m} \rightarrow t_{0} \in[0,1]$. Moreover, the compactness of $\bar{\Omega}$ and Lemma 3.3 imply that, up to a subsequence, $\beta\left(u_{m}, v_{m}\right) \rightarrow$ $y \in \bar{\Omega}$. From Lemma $3.4 \alpha_{\lambda_{m}, \delta_{m}} \rightarrow 1$. So, we can use the definition of $H_{\lambda, \delta}$ to conclude that $H_{\lambda_{m}, \delta_{m}}\left(t_{m}, u_{m}, v_{m}\right) \rightarrow y \in \bar{\Omega}$, which is a contradiction. The lemma is proved.

\section{Proof of main result}

In this section we shall prove Theorem 1.1. We begin with the following lemma.

Lemma 4.1. If $(u, v)$ is a critical point of $\mathcal{E}_{\lambda, \delta}$ on $\mathcal{N}_{\lambda, \delta}$, then it is a critical point of $\mathcal{E}_{\lambda, \delta}$ in $E$.

Proof. The proof is almost the same as that Lemma 3.2 in [15] and is omitted here.

Lemma 4.2. Suppose that $\left(F_{0}\right)-\left(F_{2}\right)$ hold, then any sequence $\left\{\left(u_{m}, v_{m}\right)\right\} \subset$ $\mathcal{N}_{\lambda, \delta}$ such that $\mathcal{E}_{\lambda, \delta}\left(u_{m}, v_{m}\right) \rightarrow c<\frac{4}{N-4}\left(\frac{S_{F}}{2^{*}}\right)^{\frac{N}{4}}$ and $\mathcal{E}_{\lambda, \delta}^{\prime}\left(u_{m}, v_{m}\right) \rightarrow 0$ contains a convergent subsequence for $\lambda, \delta>0$ if $q>2$ and $\lambda, \delta \in\left(0, \lambda^{*}\right)$ if $q=2$ for some small $\lambda^{*}>0$.

Proof. By hypothesis there exists a sequence $\theta_{m} \in \mathbb{R}$ such that $\| \mathcal{E}_{\lambda, \delta}^{\prime}\left(u_{m}, v_{m}\right)-$ $\theta_{m} \mathcal{J}_{\lambda, \delta}^{\prime}\left(u_{m}, v_{m}\right) \|_{E} \rightarrow 0$ as $m \rightarrow \infty$, where $\mathcal{J}_{\lambda, \delta}(u, v)=\left\langle\mathcal{E}_{\lambda, \delta}^{\prime}(u, v),(u, v)\right\rangle$. Thus

$$
\mathcal{E}_{\lambda, \delta}^{\prime}\left(u_{m}, v_{m}\right)=\theta_{m} \mathcal{J}_{\lambda, \delta}^{\prime}\left(u_{m}, v_{m}\right)+o_{m}(1) \text {. }
$$

Recall that

$$
\left\langle\mathcal{J}_{\lambda, \delta}^{\prime}\left(u_{m}, v_{m}\right),\left(u_{m}, v_{m}\right)\right\rangle \leq 0 \text { for all }\left(u_{m}, v_{m}\right) \in \mathcal{N}_{\lambda, \delta}
$$

If $\left\langle\mathcal{J}_{\lambda, \delta}^{\prime}\left(u_{m}, v_{m}\right),\left(u_{m}, v_{m}\right)\right\rangle \rightarrow 0$, we have

$$
\int_{\Omega}\left(\lambda\left|u_{m}\right|^{q}+\delta\left|v_{m}\right|^{q}\right) d x \rightarrow 0, \quad \int_{\Omega} F\left(u_{m}, v_{m}\right) d x \rightarrow 0 .
$$

Consequently $\left\|\left(u_{m}, v_{m}\right)\right\|_{E} \rightarrow 0$.

On the other hand, if $\left(u_{m}, v_{m}\right) \subset \mathcal{N}_{\lambda, \delta}$ it follows that

$$
1 \leq C\left(\lambda\left\|\left(u_{m}, v_{m}\right)\right\|_{E}^{q-2}+\delta\left\|\left(u_{m}, v_{m}\right)\right\|_{E}^{q-2}+\left\|\left(u_{m}, v_{m}\right)\right\|_{E}^{2^{*}-2}\right)
$$

for some $C>0$. Hence we arrive at a contradiction if $\lambda, \delta>0$ and $q>2$ or $\lambda, \delta \in\left(0, \lambda^{*}\right)$ for small $\lambda^{*}>0$ when $q=2$. Thus we may assume that 
$\left\langle\mathcal{J}_{\lambda, \delta}^{\prime}\left(u_{m}, v_{m}\right),\left(u_{m}, v_{m}\right)\right\rangle \rightarrow \ell<0$. Since $\left\langle\mathcal{E}_{\lambda, \delta}^{\prime}\left(u_{m}, v_{m}\right),\left(u_{m}, v_{m}\right)\right\rangle=0$, we conclude that $\theta_{m}=0$ and, consequently, $\mathcal{E}_{\lambda, \delta}^{\prime}\left(u_{m}, v_{m}\right) \rightarrow 0$. Using this information we have

$$
\mathcal{E}_{\lambda, \delta}^{\prime}\left(u_{m}, v_{m}\right) \rightarrow c<\frac{4}{N-4}\left(\frac{S_{F}}{2^{*}}\right)^{\frac{N}{4}} \text { and } \mathcal{E}_{\lambda, \delta}^{\prime}\left(u_{m}, v_{m}\right) \rightarrow 0,
$$

so by Lemma 2.1 the proof is completed.

Below we denote by $\mathcal{E}_{\mathcal{N}_{\lambda, \delta}}$ the restriction of $\mathcal{E}_{\lambda, \delta}$ on $\mathcal{N}_{\lambda, \delta}$.

Lemma 4.3. Suppose $N \geq 5,2 \leq q<2^{*}$ and $F$ satisfies $\left(F_{0}\right)-\left(F_{2}\right)$, let $\Lambda=$ $\min \left\{\lambda^{*}, \lambda^{* *}\right\}>0, \lambda, \delta \in(0, \Lambda)$, then $\operatorname{cat}_{\mathcal{E}_{\mathcal{N}_{\lambda, \delta}}^{m_{\lambda, \delta}}}\left(\mathcal{E}_{\mathcal{N}_{\lambda, \delta}^{m_{\lambda, \delta}}}^{m_{\lambda}} \geq\right.$ cat $_{\Omega}(\Omega)$, where $\lambda^{*}, \lambda^{* *}$ given by Lemmas 3.3 and 3.5, respectively.

Proof. Assume that $\mathcal{E}_{\mathcal{N}_{\lambda, \delta}}^{m_{\lambda, \delta}}=A_{1} \cup A_{2} \cup \cdots \cup A_{m}$, where $A_{j}, j=1,2, \ldots, m$, are closed and contractible sets in $\mathcal{E}_{\mathcal{N}_{\lambda, \delta}}^{m_{\lambda, \delta}}$, i.e., there exists $h_{j} \in C\left([0,1] \times A_{j}, \mathcal{E}_{\mathcal{N}_{\lambda, \delta}}^{m_{\lambda, \delta}}\right)$ such that

$$
h_{j}(0, z)=z, \quad h_{j}(1, z)=\vartheta \text { for all } z \in A_{j},
$$

where $\vartheta \in A_{j}$ is fixed. Consider $B_{j}=\gamma^{-1}\left(A_{j}\right), 1 \leq j \leq m$. The sets $B_{j}$ are closed and $\Omega_{r}^{-}=B_{1} \cup B_{2} \cup \cdots \cup B_{m}$. We define the deformation $g_{j}:[0,1] \times B_{j}$ by setting

$$
g_{j}(t, y)=H_{\lambda, \delta}\left(t, h_{j}(t, \gamma(y))\right)
$$

for $\lambda, \delta \in(0, \Lambda)$. Note that

$$
g_{j}(0, y)=H_{\lambda, \delta}\left(0, h_{j}(0, \gamma(y))\right)=\frac{(\beta \circ \gamma)(y)}{\alpha_{\lambda, \delta}}
$$

implies

$$
g_{j}(0, y)=\frac{\alpha_{\lambda, \delta} y}{\alpha_{\lambda, \delta}}=y \text { for all } y \in B_{j},
$$

and $g_{j}(1, y)=H_{\lambda, \delta}\left(1, h_{j}(1, \gamma(y))\right)=\beta\left(h_{j}(1, \gamma(y))\right)$ implies

$$
g_{j}(1, y)=\beta(\vartheta) \in \Omega_{r}^{+} .
$$

Thus the sets $B_{j}$ are contractible in $\Omega_{r}^{+}$. So $\operatorname{cat}_{\Omega}(\Omega)=\operatorname{cat}_{\Omega_{r}^{+}}\left(\Omega_{r}^{+}\right) \leq m$.

Proof of Theorem 1.1. Using Lemma 2.1, Lemma 2.2 and Lemma 3.2 we know that $c_{\lambda, \delta}, m_{\lambda, \delta}<\frac{4}{N-4}\left(\frac{S_{F}}{2^{*}}\right)^{\frac{N}{4}}$ for $\lambda, \delta \in(0, \Lambda)$. Moreover, by Lemma $4.2, \mathcal{E}_{\mathcal{N}_{\lambda, \delta}}$ satisfies the $(P S)_{c}$ condition for all $c<\frac{4}{N-4}\left(\frac{S_{F}}{2^{*}}\right)^{\frac{N}{4}}$. Therefore, by Lemma 4.3, a standard deformation argument implies that, for $\lambda, \delta \in(0, \Lambda), \mathcal{E}_{\mathcal{N}_{\lambda, \delta}}$ contains at least $\operatorname{cat}_{\Omega}(\Omega)$ critical points of the restriction of $\mathcal{E}_{\lambda, \delta}$ on $\mathcal{N}_{\lambda, \delta}$. Now Lemma 4.1 implies that $\mathcal{E}_{\mathcal{N}_{\lambda, \delta}}$ has at least $\operatorname{cat}_{\Omega}(\Omega)$ critical points, and therefore has at least $\operatorname{cat}_{\Omega}(\Omega)$ nontrivial solutions of (1.1). The proof is completed. 


\section{References}

[1] C. O. Alves and Y. H. Ding, Multiplicity of positive solutions to a $p$-Laplacian equation involving critical nonlinearity, J. Math. Anal. Appl. 279 (2003), no. 2, 508-521.

[2] T. Bartsch and Y. Guo, Existence and nonexistence results for critical growth polyharmonic elliptic systems, J. Differential Equations 220 (2006), no. 2, 531-543.

[3] F. Bernis, J. Garcia-Azorero, and I. Peral, Existence and multiplicity of nontrivial solutions in semilinear critical problems of fourth order, Adv. Differential Equations 1 (1996), no. 2, 219-240.

[4] H. Brezis and E. Lieb, A relation between pointwise convergence of functions and convergence of functionals, Proc. Amer. Math. Soc. 88 (1983), no. 3, 486-490.

[5] J. Chabrowski and J. Marcos do O, On some fourth-order semilinear elliptic problems in $R^{N}$, Nonlinear Anal. 49 (2002), no. 6, 861-884.

[6] L. Ding and S. W. Xiao, Multiple positive solutions for a critical quasilinear elliptic system, Nonlinear Anal. 72 (2010), no. 5, 2592-2607.

[7] D. E. Edmunds, D. Fortunato, and E. Jannelli, Critical exponents, critical dimensions and the biharmonic operator, Arch. Rational Mech. Anal. 112 (1990), no. 3, 269-289.

[8] D. C. de Morais Filho and M. A. S. Souto, Systems of p-Laplacian equations involving homogeneous nonlinearities with critical Sobolev exponent degrees, Comm. Partial Differential Equations 24 (1999), no. 7-8, 1537-1553.

[9] F. Gazzola, H.C. Grunau, and M. Squassina, Existence and nonexistence results for critical growth biharmonic elliptic equations, Calc. Var. Partial Differential Equations 18 (2003), no. 2, 117-143.

[10] Y. X. Ge, J. C. Wei, and F. Zhou, A critical elliptic problem for polyharmonic operators, J. Funct. Anal. 260 (2011), no. 8, 2247-2282.

[11] H. Grunau, Positive solutions to semilinear polyharmonic Dirichlet problems involving critical Sobolev exponents, Calc. Var. Partial Differential Equations 3 (1995), no. 2, $243-252$.

[12] P. G. Han, The effect of the domain topology on the number of positive solutions of an elliptic systems involving critical Sobolev exponents, Houston J. Math. 32 (2006), no. 4, 1241-1257.

[13] T. S. Hsu and H. L. Lin, Multiple positive solutions for a critical elliptic system with concave-convex nonlinearities, Proc. Roy. Soc. Edinburgh Sect. A 139 (2009), no. 6, 1163-1177.

[14] D. S. Kang and S. J. Peng, Existence and asymptotic properties of solutions to elliptic systems involving multiple critical exponents, Sci.China Math. 54 (2011), no. 2, 243-256.

[15] D. F. Lü, Multiple solutions for a class of biharmonic elliptic systems with Sobolev critical exponent, Nonlinear Anal. 74 (2011), no. 17, 6371-6382.

[16] D. F. Lü and J. H. Xiao, Multiple solutions for weighted nonlinear elliptic system involving critical exponents, Math. Comput. Modelling 55 (2012), no. 3-4, 816-827.

[17] M. Montenegro, On nontrivial solutions of critical polyharmonic elliptic systems, J. Differential Equations 247 (2009), no. 3, 906-916.

[18] E. S. Noussair, C. A. Swanson, and J. Yang, Critical semilinear biharmonic equations in $R^{N}$, Proc. Roy. Soc. Edinburgh Sect. A 121 (1992), no. 1-2, 139-148.

[19] O. Rey, A multiplicity results for a variational problem with lack of compactness, Nonlinear Anal. 13 (10) (1989), no. 10, 1241-1249.

[20] Y. Shen and J. H. Zhang, Multiplicity of positive solutions for a semilinear p-Laplacian system with Sobolev critical exponent, Nonlinear Anal. 74 (2011), no. 4, 1019-1030.

[21] Y. J. Wang and Y. T. Shen, Multiple and sign-changing solutions for a class of semilinear biharmonic equation, J. Differential Equations 246 (2009), no. 8, 3109-3125.

[22] M. Willem, Minimax Theorems, Birkhäuser, Boston, 1996.

[23] Y. J. Zhang, Positive solutions of semilinear biharmonic equations with critical Sobolev exponents, Nonlinear Anal. 75 (2012), no. 1, 55-67. 
Dengfeng Lü

School of Mathematics and Statistics

Hubei Engineering University

Xiaogan, Hubei 432000, P. R. China

E-mail address: dengfeng1214@163.com

JiAnhai XiaO

School of Mathematics and Statistics

Hubei Engineering University

Xiaogan, Hubei 432000, P. R. China

E-mail address: jhxmath@sina.cn 